\title{
Using LoRaWAN Technology To Enhance Remote Power Network Monitoring
}

\author{
Ross McPherson, Craig Hay, James Irvine, Senior Member, IEEE
}

\begin{abstract}
Future power networks will require much greater levels of machine-to-machine interaction to enable smart grid applications, and ultimately increase the efficiency of the power network. Low-Power Wide-Area Network (LPWAN) technology enables the deployment of many new IoT solutions in areas such as controlling and monitoring assets, environmental sensing and location tracking. In this paper, a prototype low-cost LPWAN module is developed to be retrofitted to existing Fault Passage Indicators, creating a automatic remote detection and location system for medium voltage faults, reducing the time to find faults from hours to minutes. This prototype device was then tested on a physical $11 \mathrm{kV}$ overhead line network, where the device demonstrated it was robust and suitable for mass deployment on a live network. The finalised prototype module costs roughly $5 \%$ of a single FPI unit, and additionally features the ability to remotely reconfigure FPI making installation and maintenance more convenient.
\end{abstract}

Index Terms-Smart Grid, Power, Communications, Automation, Product Development, Security, Power Transmission Faults

\section{INTRODUCTION}

I NCREASING the efficiency of the power network, through the application of smart grids, will require a much greater level of machine-to-machine interaction. Low-Power WideArea Network (LPWAN) technology enables the deployment of many new IoT solutions in areas such as controlling and monitoring assets, environmental sensing and location tracking. Although much of this has been technically possible previously, it was not cost effective. LPWAN makes such deployments viable particularly in rural areas where assets are geographically distant, and therefore existing technologies such as Bluetooth Low Energy or Zigbee, would have required a lot of supporting infrastructure, due to their relatively short range.

\section{LPWAN TECHNOLOGY}

LPWAN technology is best suited for power constrained devices used in applications requiring long-range communications, and thousands of connected devices. End-devices in an LPWAN are typically battery-powered, which makes deployment notably easier, since there is no requirement to find an external power source. In addition, end-devices can also be systematically provisioned, are low cost and typically are being characterised as low throughput, mainly idle devices that can receive limited downstream traffic. All of these

The authors are with the Department of Electronic \& Electrical Engineering, University of Strathclyde, Glasgow, G1 1XW, UK. Email: craig.hay@strath.ac.uk characteristics produce a package suitable for remote sensing and monitoring.

LoRaWAN is a LPWAN communications protocol which, using its supporting physical layer - LoRa - provides the functions of Layer 2 of the OSI reference networking stack, constructing a straightforward environment for devices to communicate back to a control centre. This is achieved within the LoRaWAN architecture by an end-device transmitting data over radio-waves on the $868 \mathrm{MHz}$ frequency to a LoRaWAN gateway, which then forwards messages to a remote server, either via $4 \mathrm{G}$ mobile communications or via a wired IP network. Multiple gateways can be deployed to pass messages back to the same server in a star-of-stars network topology, creating a robust network where several gateways can receive and pass along the message.

Sigfox is a technology standard which offers an LPWAN physical radio infrastructure along with supporting network architecture. By adding a low cost addon Sigfox module to a design and paying the subscription fee, all messages send from the device will be delivered automatically to the end user via a number of provided API endpoints. These endpoints can then feed into companies existing systems or be integrated with commonly used presentation dashboards. In line with LoRaWAN, Sigfox also transmits over unlicensed spectrum which reduces the cost of deployment since spectrum does not have to be procured.

NB-IoT is a further LPWAN technology, which differs from both LoRaWAN and Sigfox in that it utilises existing mobile network infrastructure and transmits within licensed frequency bands. On the other hand it also retains functional similarities with both LoRaWAN and Sigfox, but the subscription model is more closely aligned with Sigfox. The end user cannot affect network coverage and simply joins their device to an existing network, and the messages are directly delivered to the end user.

Due to the recent emergence of commercial providers of LoRaWAN networks who deliver infrastructure for a fee, similar to Sigfox and traditional cellular network providers, there are now many available options for deploying LoRaWAN. These options are the utility subscribing to use a third party network and physical infrastructure, the utility building its own network and infrastructure independent from any other company, or the utility employing a hybrid approach, combining the coverage from a commercial provider with utility owned infrastructure used to fill coverage gaps. Being able to fill coverage gaps is important, as the unfavourable location of the utility assets often makes it economically unfeasible for telecommunication companies to provide network coverage in 


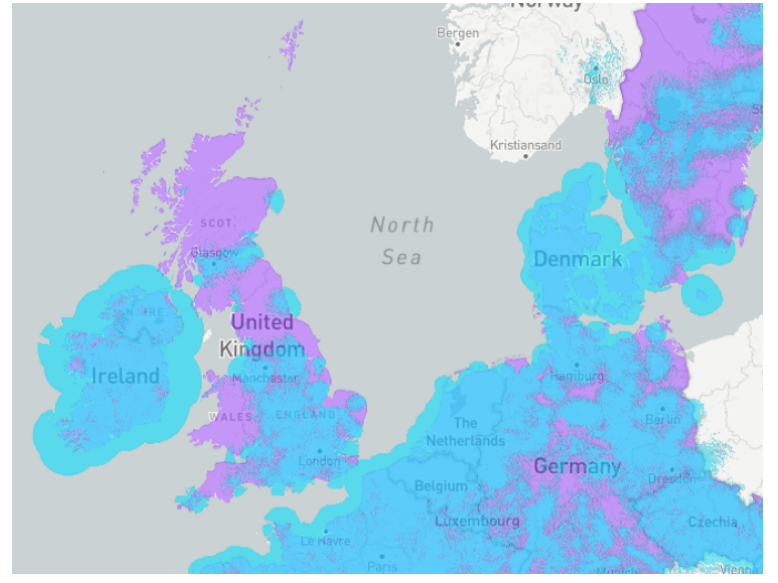

Fig. 1: Sigfox Coverage Map [1]

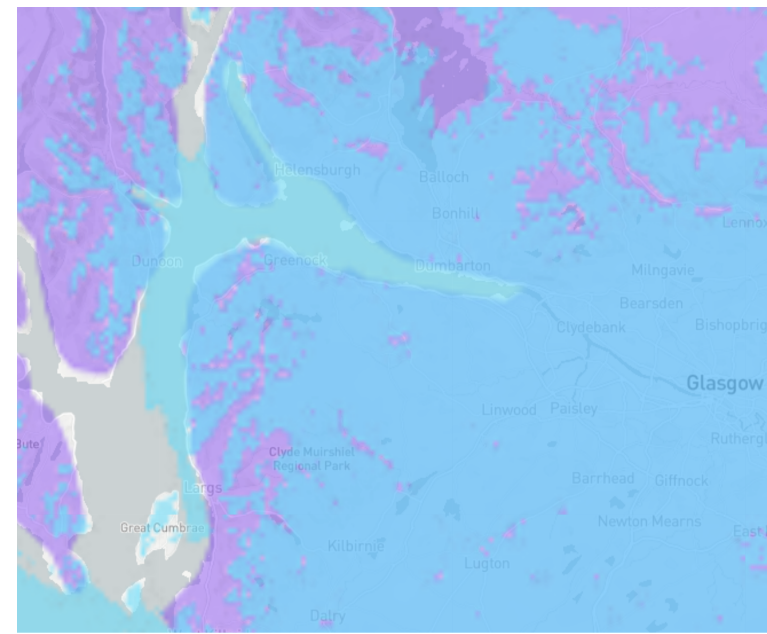

Fig. 2: Sigfox Coverage Map - Around Glasgow [1]

those areas. For example, figure 1 shows the current Sigfox coverage in the UK. The lighter areas of the map denote areas of coverage, whereas the darker areas indicate a lack of coverage. As shown in figure 1 , the overall coverage is sparse, and there is a total absence of coverage outside of major population centres, making Sigfox unsuitable for rural environments. Furthermore, gaps in coverage become even more apparent upon focusing on a particular area, as shown in figure 2. The coverage around the city of Glasgow is good, but patchy on the surrounding islands (presumably due to the terrain), making Sigfox currently unsuitable for a reliable island wide deployment.

\section{TEST CASE}

In order to test the feasibility of LPWAN technology within the power industry, an existing sensor device was integrated with LPWAN communications. Given the low rural coverage of Sigfox and, at the time, limited implementation of NBIoT, LoRaWAN was selected for the communications and a fault passage indicator (FPI) was selected as the sensor device. Fault passage indicators are used to give an indication of the passage of fault current along a section of cable and assist in the isolation of overhead line faults in rural areas. Medium voltage faults account for a significant number of customer minutes lost per year. Traditional fault passage indicators have a physically visible detection indicator, either a mechanical flag or a light, to indicate the detection of fault current on the line [2].

In the event of a fault, to utilise the information given by the indicator, the first step is to walk or drive along the length of the line to see which FPIs are indicating the recent passage of fault current. Typically this can take hours to complete, depending on the topology of the line and the geography of the area it traverses. Upgrading FPIs to use wireless communications which report the passage of fault current can dramatically reduce the time taken to determine the location of a fault to a few minutes, as personnel are no longer required to traverse the length of the line.

\section{Previous Work}

Fault passage indicators with cellular communications have existed for a number of years. Unfortunately these do not suit all locations, as many of the areas where FPIs are deployed suffer from poor cellular coverage due to their rural location. It makes little economic sense for telecommunications providers to guarantee coverage in those areas due to very low population density. In such scenarios, Distribution Network Operators (DNOs) have seen positive results from installing other wireless technologies to provide connectivity [3]. A similar scheme of installing thousands of FPIs with remote monitoring was said to reduce the length of outages at Liander - the Dutch utility operator - by half an hour [4]. This resulted in a reduction in Customer Minutes Lost during an outage, decreasing fines for the network operator and increasing customer satisfaction.

Carrillo \& Seki investigated the rural deployment of IoT devices and the infrastructure which would be required to provide coverage, one of the technologies within their study was LoRaWAN. They found that increasing the height of the LoRaWAN gateway increased the coverage [5]. This finding along with supporting evidence from Carvalho Silva et al [6], suggest that within rural environments a range of $45 \mathrm{~km}$ is achievable.

\section{DeVelopment}

The FPI integrated with LoRaWAN radio (hereinafter referred to as the LoRaWAN-FPI device) was developed by initially connecting the UART serial bus on the chosen FPI (Bowdens Pathfinder 360 Alpha) to a PC via a USB-serial converter in order to test the available command set. The manufacturer of the FPI helpfully provided a document detailing the protocol used for UART communication, a modified Hayes command set, which eased the development effort. The next stage was to design a PCB module, which would feature a LoRaWAN module to send and receive messages, and a microcontroller to act as an intermediary between the FPI and LoRaWAN module. The microcontroller software converted long, verbose FPI messages into short LoRaWAN reporting messages, provided heartbeat messages, and formed the verbose serial commands required to reconfigure the FPI. 


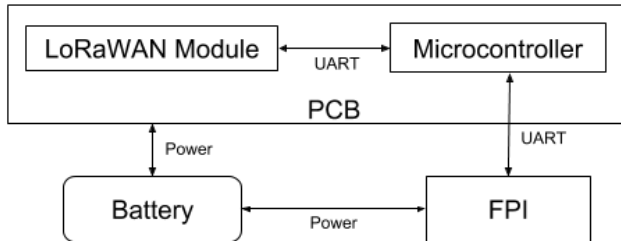

Fig. 3: LoRaWAN-FPI Design Layout

This was achieved by using two UART connections on the microcontroller, one to communicate with the FPI and the other with the LoRaWAN module, as shown in figure 3. Additionally, as the solution utilises the existing UART connections on the FPI and a 3.7V DC supply from the FPI's long life lithium thionyl chloride battery, currently deployed FPIs could be retrofitted if desired.

\section{A. Iterative Testing}

During the software development phase the device underwent extensive iterative testing in the lab. By altering the surrounding magnetic field (using a small permanent magnet), the FPI could be triggered. This process effectively emulates the change in magnetic field detected when fault current passes on an overhead power line. The triggered fault could then be used to modify the code as required, and ensure robustness since the module was designed to be a deploy and forget solution.

\section{B. Practical Concerns}

One area of concern while developing the device was how to supply power. Although the device would be situated underneath a power line, in practice it is expensive and impractical to step down the voltage to usable levels for the FPI. In addition to this the device would require a back-up battery to power it when a fault has occurred. Therefore, the device had to run on battery power and last for a number of years, so while designing the system care was taken to ensure it would be energy efficient, this was partially achieved by using dedicated low energy consumption components, and partially by programming the microcontroller to enter low power sleep mode until triggered by the FPI. Another concern was keeping the add-on module low cost so that it would be practical to produce and deploy on a large scale. This was regarded as successful considering the cost of producing add-on module would be approximately $£ 15$ at a scale of 2000 units.

It was decided to power the LoRaWAN module using the battery included in the FPI, since it was originally designed to last for 10 years while powering the FPI circuitry and a GSM communications module. Furthermore, LoRaWAN consumes less power than GSM, and the expectation was that using LoRaWAN would significantly reduce the time to find the fault on the network (shortening the period the FPIs power intensive LED would be flashing and further reducing power consumption).

\section{Joining}

Another programming design decision was to automatically attempt to try and connect to the LoRaWAN network once the device received power, and if the device failed to connect or attempted to transmit a message while not being connected to the network. The device would periodically attempt to rejoin the network and upon successful connection would transmit any backlogged messages. This means that if the LoRaWAN network was non-operational, the physical device would not need to be reset to become functional again, which would involve unmounting every device from its pole in a remote area and power cycling each device individually.

\section{Reconfiguration}

Once the initial program design was completed, it was decided to expand the design to include remotely re-configuring the FPI over LoRaWAN using downlink messages. This was achieved by using a feature of LoRaWAN class A devices, where upon successful completion of sending an uplink message the device will listen for a short window of time for a response from the gateway. This response can either be an acknowledgement of the successful receipt of the message, or a message stored on the central server waiting to be delivered to the LoRaWAN device. Although this procedure is high latency, as messages can only be delivered after the device transmits, it does greatly decrease the battery consumption of the end-device, considering the device does not have to listen permanently for messages. For the use case of LoRaWAN enabled FPIs this delay is not a significant inconvenience, partly due to if the device is misconfigured and triggering prematurely it will frequently be sending messages. However, even if a heartbeat message is only configured to be once per day, that is still more efficient than manually taking down the FPI, taking it back to a workshop to be opened up finally redeployed.

\section{E. Web Interface}

A Web interface was also developed which, integrates with the LoRaWAN Application Server to display any faults on a map so they can be found quickly and easily, reducing the number of customer minutes lost when a fault occurs. This web interface was additionally designed to integrating with DNOs existing and future computer systems, for example, if the utility wanted to utilise an existing monitoring dashboard, or create an accompanying mobile application so that field technicians could be alerted and be routed to the issue.

\section{TESTING}

To complement the lab testing, the LoRaWAN-FPI device was also tested on an isolated $11 \mathrm{kV}$ trial network to prove the constructed device would correctly operate on a real power network. The $11 \mathrm{kV}$ network testing involved introducing faults onto the electrical network, while observing and verifying both the traditional visual response of the FPI and the LoRaWAN remote reporting. 


\section{A. Test Plan}

To confirm the LoRaWAN-FPI would operate as planned, a subsection of the University of Strathclyde Power Networks Demonstration Centre's (PNDC) $11 \mathrm{kV}$ network was dedicated to testing the device. Initial tests were designed to affirm the standalone FPI operated correctly, followed by confirming that faults thrown on the network would be accurately reported back to the central control centre over LoRaWAN. Finally, the most crucial test was to certify the reconfiguration of the LoRaWAN-FPI had correctly altered the magnitude of fault current required to trigger the device. This was the most important since lab tests could not readily confirm the successful reconfiguration of the FPI, as the reconfigurable parameters of the device were so slight it was challenging to emulate the change required using only permanent magnets.

As mentioned in section $\mathrm{V}-\mathrm{C}$ the device configured to automatically join a LoRaWAN network upon receiving power, if this network was not available or nonfunctional the device would periodically check and try to connect again, therefore once the device was powered it would connect without external involvement. The LoRaWAN network in question was provided interchangeably by one of the PNDC LoRaWAN gateways. For testing purposes one was connected via a 3rd party ADSL line and the second was connected via the $4 \mathrm{G}$ cellular network, since both types of IP communcations cover the most common types of connections which would be provided when deploying a LoRaWAN network. Both of these gateways were configured to forward LoRaWAN messages onto the web interface mentioned in section V-E so that incoming messages could be easily visualised, showing simply if it was a heartbeat message, an instantaneous fault or a permanent fault.

\section{B. Test Setup}

A subsection of the PNDC $11 \mathrm{kV}$ network was used to test the LoRaWAN-FPI, the testing utilised the PNDC HV network powered by the motor-generator set, and the HV fault thrower. The circuit was loaded using load banks, and the fault thrower first threw a fault generating a fault current of $15 \mathrm{~A}$. In the event of an unsuccessful attempt at detecting the fault using the FPI, the fault was to be thrown again until successfully detected. A successful detection would be confirmed by observing the flashing white LED on the FPI. The LoRaWAN-FPI itself was physically mounted upon a standard $11 \mathrm{kV}$ utility pole, between a mock impedance unit and a pole mounted voltage regulator. Substations A, D and G were used to apply maximum loading to the network. Initially, only a simple fault was to be tested to confirm it was operating correctly, and then the FPI was reset to be ready for the next test.

To confirm reconfiguration of the FPI over the LoRaWAN network, the FPI was issued a command to increase the fault current threshold. Then the fault resistance used in the HV fault thrower was modified to put more energy into the fault. table I lists the fault types and associated resistances that were applied during testing. These values were selected such that maximum and minimum fault current conditions of the FPI were used. Between each fault thrown the FPI and the network

\begin{tabular}{|l|l|l|l|}
\hline Fault ID & Fault Type & Fault Resistance & Fault Current \\
\hline F1 & Phase to Earth & 240 & $15 \mathrm{~A}$ \\
\hline F2 & Phase to Earth & 20 & $50 \mathrm{~A}$ \\
\hline
\end{tabular}

TABLE I: Summary of fault parameters

was reset to ensure results were not effected by any residual elements.

Finally, the system was reset once more and the system was left to run in normal operation to confirm the LoRaWAN-FPI would periodically check-in using heartbeat messages while deployed.

\section{Test Results}

For all tests shortly after a fault was thrown the device would illuminate, demonstrating the FPI acknowledged the fault. Then the web interface would detail the type of fault experienced, along with the time of the fault, and the location of the device in question, demonstrating the successful transmission of faults via the LoRaWAN network, while on an $11 \mathrm{kV}$ network.

During the reconfiguration procedure, a permanent fault was thrown at $15 \mathrm{~A}$ and the FPI successfully triggered on the first attempt. The FPI was then reconfigured over the LoRaWAN network to trigger at its highest fault level of 45A. The HV network was reset, and the previous fault of $15 \mathrm{~A}$ was thrown. The FPI did not trigger, as indicated by no illumination. The $\mathrm{HV}$ fault thrower was reconfigured again to throw a fault of 50A and the FPI triggered successfully. This sequence of tests proved that the FPI was successfully reconfigured over the LoRaWAN network.

The amount of data received by and transmitted from the FPI also was recorded, and found to be not significant although the most significant factor in data usage would be how frequently the operator would like their FPIs to check-in with a heartbeat message, which could report battery level and check if any FPI reconfiguration has been requested. It is worth noting more frequent messages would decrease the battery life and potentially increase costs as future commercial LoRaWAN networks could bill users for the data they use, if the utility choice to using an external LoRaWAN network supplier, rather than deploying their own network. Barring exceptional circumstances the devices should not require reconfiguration or battery checks frequently, e.g. once every two weeks. The size of the LoRaWAN packets used within this test from the FPI were a consistent 16 bytes, 14 bytes of LoRaWAN overhead and 2 bytes of payload. The downlink packets to reconfigure the FPI were 22 bytes, as 8 bytes were required to encode all of the FPI reconfiguration parameters.

The latency of the communications was also measured by recording the time faults were thrown and the time messages were received by the LoRaWAN Application Server. This was found to be 30 seconds, due to the fault detection procedure followed by the internal logic of the FPI, where it waits for potential upstream autorecloser operations to complete before signalling a permanent fault. This means there is a delay before passing the message along to the microcontroller via the UART, where the microcontroller can then action it and 
send it over LoRaWAN. The latency from sending a message from the LoRa module to the web interface was found to be within a couple of seconds.

\section{IMPLICATIONS FOR DEPLOYMENT}

This proof of concept has combined two technologies high on the technology readiness level (TRL) scale by creating a custom add-on module, which was tested and confirmed to operate as expected. Therefore it has shown that for this use case, the remote detection of fault currents, LoRaWAN can meet all of the requirements. Even given this success, there are many practicalities of deployment that still have to be considered.

\section{A. Deployment Strategy}

Since a gateway is required to receive the messages from end-devices and pass them to the servers, and the cost of an outdoor gateway being around $£ 1000$ plus the cost of procuring thousands of add-on LoRaWAN boards, this may seem like a large capital investment. On the other hand utilities spend on average $£ 20 \mathrm{k}$ on a single remote terminal unit (RTU), therefore in comparison the capital outlay is not significant. Furthermore as previously mentioned in section V-B the cost of the LoRaWAN PCB add-on boards is small compared to the price of the FPI unit.

On the contrary, if the area is a low density rural area with requiring few sensors, it would prove costly per sensor to roll-out a LoRaWAN solution unless a low cost gateway can be procured or developed. Moreover, eight channel gateways are not low power devices, consuming approximately $50 \mathrm{~W}$, so require a DC power source typical of other networking equipment. The gateway also needs to be situated in an area with either cellular coverage or wired (IP based) connectivity in order to communicate with the network server. Meeting these requirements in rural areas could require the installation of equipment to provide LV power tapped from $\mathrm{HV}$ lines further increasing the cost per sensor deployment.

\section{B. Retrofitting}

DNOs own a lot of existing network equipment that will not be replaced for many years. If LPWAN technology solves a business problem and a mass deployment is shown to be beneficial, widespread deployment would require the retrofitting of new LPWAN hardware to existing equipment. This brings about challenges on the technical and logistical front. Battery powered 'fit and forget' sensing devices can reduce the cost of deployment by around $80 \%$. With careful management of power and infrequent data transmissions, a LoRaWAN Class A device can be expected to run for between 1-5 years on a small primary battery. Energy harvesting could potentially extend this lifetime, in some cases indefinitely.

\section{Rural Areas}

Due to the size of the facilities at PNDC, the RF parameters of the LoRaWAN network were not tested, however, as detailed in section IV research has been conducted into this area. The surrounding area is rural, and further trials may take place with end-devices positioned at remote locations in the area around PNDC. Local substations would be the natural fit for future locations.

\section{City Environments}

City environments are more of a challenge for network deployment due to the proximity of buildings. This creates a complex multipath environment for the signals between end-device and gateway. Although LoRaWAN does provide good coverage in buildings compared to other competing technologies, [7] the range in urban environments has been reported to be around $5 \mathrm{~km}$, although this is highly variable. [8] Therefore, care should be taken when deploying a LoRaWAN network in an urban area depending on the geography.

\section{CONCLUSION}

LoRaWAN was a proved to be an effective communication mechanism for low data rate, predominately sensor to server communication. As evidenced this arrangement works well for fault passage indicators due to the infrequent messages required. The created device was thoroughly tested, both in the laboratory and on an physical power network, which proved the device would operate correctly when deployed. Additionally the ability to retrofit existing FPIs significantly reduces the deployment cost, as the retail cost of the add-on module would be roughly $12 \%$ of the cost of the FPI. Moreover, these retrofitted devices can be left for unmaintained for years, as its self diagnosing and rectifying code means the created device is robust enough to left unattended. Additionally, the facility to remotely reconfigure FPIs dynamically will make installing and maintaining these devices easier. All of these represent significant advantages to network operators while most importantly the device will reduce the time to find faults from hours to minutes, and these faults will be automatically reported without relying on human reports.

\section{REFERENCES}

[1] (2018, March). [Online]. Available: https://www.sigfox.com/en/coverage

[2] T. Newman, "Pole mounted fault passage indicators," in IEE Colloquium on Improving Supply Security on 11 kV Overhead Networks, May 1990, pp. $7 / 1-7 / 2$.

[3] J. W. Knapek, S. Gamache, and J. Fowler, "Communications-capable fault indicators improve outage response for coastal oregon," in 2016 IEEE Rural Electric Power Conference (REPC), May 2016, pp. 91-95.

[4] S. Hodgson, "The use gsm and web based scada for monitoring fault passage indicators," in IEEE PES T D 2010, April 2010, pp. 1-3.

[5] D. Carrillo and J. Seki, "Rural area deployment of internet of things connectivity: Lte and lorawan case study," in 2017 IEEE XXIV International Conference on Electronics, Electrical Engineering and Computing (INTERCON), Aug 2017, pp. 1-4.

[6] J. de Carvalho Silva, J. J. P. C. Rodrigues, A. M. Alberti, P. Solic, and A. L. L. Aquino, "Lorawan: A low power wan protocol for internet of things: A review and opportunities," in 2017 2nd International Multidisciplinary Conference on Computer and Energy Science (SpliTech), July 2017, pp. 1-6.

[7] J. Haxhibeqiri, A. Karaagac, F. V. den Abeele, W. Joseph, I. Moerman, and J. Hoebeke, "Lora indoor coverage and performance in an industrial environment: Case study," in 2017 22nd IEEE International Conference on Emerging Technologies and Factory Automation (ETFA), Sept 2017, pp. $1-8$.

[8] N. Blenn and F. Kuipers, "Lorawan in the wild: Measurements from the things network," arXiv preprint arXiv:1706.03086, 2017. 